Albitzky: Kenntnis von Isomerieverhältnissen etc. 289

Untersuchungen aus dem chemischen Laboratorium von Prof. Alexander Saytzeff za Kasan.

\title{
89. Beitrag zur Kenntnis von Isomerieverhältnissen der Öl-, Elaidin-, Eruka- und Brassidinsänre;
}

von

\section{Alexius Albitzky.}

\section{( $Z$ weite Abhandlung.)}

Im 61 . Bande dieses Journals ${ }^{1}$ ) habe ich gezeigt, daB bei nachfolgender Einwirkung von unterchloriger Säure und Ätzkali auf Öl-, Elaidin-, Eruka- und Brassidinsäure Dioxysäuren erhalten werden, jedoch nicht jene, die aus der betreffenden ungesättigten Säure bei ibrer Oxydation durch Kaliumpermanganat resultieren, sondern aus einer anderen stereoisomeren Reihe. So erhält man aus Ölsäure Dioxystearinsäure vom Schmelzp. 99,5 $5^{\circ}$, aber nicht die vom Schmelzp. 136,5 wie zu erwarten gewesen wäre u. s. w.

Bei weiteren Untersuchungen in dieser Richtung ergab sich, daß genannte Reaktion nicht die einzige ist, bei der vom Gesichtspunkt der geometrischen Isomerie anormale Produkte erhalten werden, sondern daß man auch bei anderen Reaktionen aus einer gegebenen ungesättigten Säure nach $W$ unsch die eine oder andere Dioxysäure erhalten kann, dab man eine fertige Dioxysäure in eine andere überführen kann, die ihr stereoisomer ist, und schließlich daß man von einer Dioxysäure zu einer ungesättigten der anderen stereoisomeren Reihe übergehen kann.

Aus Versuchsresultaten kann geschlossen werden, daß es nicht immer möglich ist, aus der Konfiguration des Ausgangsproduktes die Konfiguration des erhaltenen Produktes zu folgern, da, wie im gegebenen speziellen Falle, auch die Bedingungen, unter denen die Reaktion vorgenommen wird,

1) Seite $65-94$.

Journal f. prakt, Chemio [2] $\mathrm{Bd}_{\mathrm{r}} 67$. 
Bedeutung gewinnen, z. B. ob die Lösung sauer oder alkalisch ist. Diesen Gedanken eines Einflusses der Art des Reagens auf die Natur des resultierenden Produktes (im Sinne der Stereoisomerie) sprach ich in einer vorläufigen Mitteilung ${ }^{1}$ ) aus, und er findet Unterstützung in folgenden Worten des bekannten amerikanischen Chemikers A rthur Michael: „Solche unlösbare Widersprüche haben ihren Grund hauptsächlich darin, daß der stereochemische Verlauf beim Übergang von ungesättigten zu gesättigteren Körpern, sowie bei den umgekehrten Prozessen von den in Tätigkeit tretenden chemischen Kräften abhängt, und daher nicht nur eine Funktion der im Moleküle vorhandenen Atome, sondern auch des angreifenden Reagens ist. "2)

$\mathrm{Da}$ schon gefunden worden ist, daß unter gewissen Bedingungen die Möglichkeit eintritt, aus einer ungesättigten Verbindung eine gesättigte $\mathrm{zu}$ erhalten, die als anormales Produkt der Reaktion auftritt, so war es interessant, zu untersuchen, ob und unter welchen Bedingungen man von einer gesättigten Verbindung, im gegebenen Falle einer Dioxysäure, zu einer ungesättigten Säure übergehen kann, und zwar so, daB letztere auch als anormales Produkt der Reaktion erscheine, d. h. dab sie zur anderen stereoisomeren Reihe gehöre. Außerdem war es interessant zu erfahren, ob die Umwandlung einer Dioxysäure in ihr Stereoisomeres möglich ist, ähnlich den Fällen, wo man eine ungesättigte Säure in ihre stereoisomere überführen kann.

Umwandlung der Dioxystearinsäure vom Schmelzpunkt $136,5^{\circ}$ in die Elaidinsäure.

Zur Lösung der ersten Frage wurden Versuche der Um. wandlung einer Dioxystearinsäure in Dibromstearinsäure mit Hilfe von $\mathrm{HBr}$ unternommen:

$$
\mathrm{C}_{18} \mathrm{H}_{38}(\mathrm{OH})_{2} \mathrm{COHO}+2 \mathrm{HBr}=\mathrm{C}_{17} \mathrm{H}_{38} \mathrm{Br}_{2} \mathrm{COHO}+2 \mathrm{H}_{2} \mathrm{O} \text {. }
$$

Dem Bromprodukt wurde sodann das Brom entzogen.

Versuch I. $10 \mathrm{~g}$ einer Dioxystearinsäure vom Schmelzpunkt $133^{\circ}-135^{\circ}$, zu feinstem Pulver zerrieben, wurden in

1) Ber. 33, 2910 (1900).

2) Das. 34, 3646 (1901). 
Albit/ky: Kenntnis von Isomerieverhältnissen etc. 291

einen Kolben, mit ein wenig Wasser angerührt, mit gasförmigem Bromwasserstoff unter Kühlung mit Eiswasser und öfterem Umschwenken des Kolbens gesättigt. Bromwasserstoff wurde aus Bromkalium und Schwefelsäure gewonnen ( 1 T. $\mathrm{H}_{2} \mathrm{O}$ und $3 \mathrm{~T} . \mathrm{H}_{2} \mathrm{SO}_{4}$ ); behufs Reinigung wurde er durch eine leere, mit Eiswasser gekühlte Drechselsche Flasche geleitet, in der die Wasserdämpfe sich kondensierten, wonach er noch durch eine mit rotem Phosphor und Glasscherben gefüllte U-förmige Röhre geführt wurde. Beim Sättigen mit Bromwasserstoff verwandelte sich die Dioxystearinsäure allmählich in eine dicke, zähflüssige, braunrote, ölige Flüssigkeit, in der sehr wenig Stückchen Dioxystearinsäure enthalten waren, welche bis zum nächsten Tage auch fast alle verschwanden. Nachher wurde der Kolben eine Zeitlang auf dem Wasserbade erwärmt. SchlieBlich wurde nach dem Erwärmen in den Kolben Wasser hinzugesetzt, und das Bromprodukt mit Äther ausgezogen.

Analyse dieses Bromürs:

0,258 g Substanz gaben 0,223 $\mathrm{g}$ AgBr.

$$
\begin{array}{ccc} 
& \text { Berechnet für } \mathrm{C}_{18} \mathrm{H}_{34} \mathrm{Br}_{2} \mathrm{O}_{2} \text { : } & \text { Gefunden: } \\
\mathrm{Br} & 36,16 & 36,78 \%
\end{array}
$$

Um das Brom zu entfernen, wurde das Bromür in absoIutem Alkohol gelöst, diesem Zinkspäne und Salzsäure hinzugefügt; die Reaktion wurde anfangs bei gewöhnlicher Temperatur, später auf dem Wasserbade ausgeführt. Nach Beendigung der Reaktion wurde die Flüssigkeit abfiltriert, derAlkohol abdestilliert, und der Rückstand mit Wasser ausgekocht. Die abgeschiedene ölige Schicht erstarrte beim Erkalten. Um die bei dieser Reaktion möglicherweise entstandenen Äthylester zu zerlegen, wurde das Produkt mit Ätzkali verseift. Die durch $\mathrm{HCl}$ ausgeschiedenen Säuren, $7 \mathrm{~g}$ an Gewicht," schmolzen bei $53^{\circ}-83^{\circ}$ und erstarrten bei $42^{\circ}-40^{\circ}$. Durch mehrmaliges Umkristallisieren aus Alkohol und Äther gelang es zwei Fraktionen auszuscheiden, die bestimmte Schmelzpunkte besaBen: die eine bei $130^{\circ}-131^{\circ}$ (Erstarrungsp. 121 ${ }^{\circ}-116^{\circ}$ ), die andere bei $44^{\circ}-45^{\circ}$ (Erstarrungsp. $41^{\circ}-40^{\circ}$ ) schmelzend. Die erste Fraktion vom Schmelzp. 130 $130^{\circ}-131^{\circ}$ ist unveränderte Dioxystearinsäure, die als Beimischung beim Bromür vorhanden war; wenn trotzdem die Bestimmung des Broms ein befriedigendes 
Resultat ergab, so kann das dadurch erklärt werden, daß die Bromwasserstoffsäure aus dem Bromür nicht vollständig ausgewaschen war. Die Fraktion vom Schmelzp. $44^{\circ}-45^{\circ}$ erinnerte in ihrem Aussehen - beim Umkrystallisieren aus Äther Blättchen - an Elaidinsäure. Um solches zu beweisen, wurde aus dieser Fraktion ein Dibromid dargestellt, zu welchem Zwecke zur Benzollösung der Säure beim Erkalten tropfenweise eine Benzollösung von Brom, bis zum Entstehen der beständigen Färbung, hinzugesetzt wurde. Nach Abdampfen des Benzols und Umkristallisieren aus Äther schmolz das Dibromid bei $26^{\circ}-28^{\circ}$ und erstarrte bei $14^{\circ}-12^{\circ}$. Das Dibromid der Elaidinsäure schmilzt bei $27^{\circ}$.

$0,302 \mathrm{~g}$ Substanz gaben 0,256 g AgBr.

$$
\begin{gathered}
\text { Brechnet für } \mathrm{C}_{18} \mathrm{H}_{34} \mathrm{Br}_{2} \mathrm{O}_{2}: \quad \text { Gefunden: } \\
36,16
\end{gathered}
$$

Die Tatsache der Bildung von Elaidinsäure veranlaBte eine genauere Untersuchung dieser Reaktion, um zu bestimmen, ob hier eine volle Umlagerung der Dioxystearinsäure bei Entstehung des Dibromids stattfindet, oder ob solche nur in einem Teile derselben vor sich geht und infolge dessen zu erwarten wäre, daß unter anderen Produkten der Reaktion auch ein normales, d. h. Ölsäure sich findet.

$\mathrm{Zu}$ diesem Zwecke wurde derselbe Versuch einer Umwandlung der Dioxystearinsäure in Dibromstearinsäure, nur in größerem Maßstabe, wiederholt.

Versuch II. Alle Operationen wurden genau ebenso ausgeführt mit $57 \mathrm{~g}$ einer Dioxystearinsäure, wie im Versuch I. Infolge länger fortgesetzter Sättigung mit Bromwasserstoff und länger anhaltendem Erwärmen auf dem $W$ asserbade, zwecks Erzielung eines gleichartigen Produktes, nahm dasselbe eine fast schwarzbraune Färbung an und wurde bedeutend dickflüssiger als bei Versuch $I$.

$0,2555 \mathrm{~g}$ Substanz gaben $0,1685 \mathrm{~g}$ AgBr.

$$
\begin{aligned}
& \text { Berechnet für Gefunden: }
\end{aligned}
$$

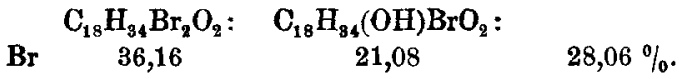

Die Analyse zeigt, daB das hierbei resultierte Dibromid nicht rein ist; dieses kann entweder durch Anwesenheit von 
nicht umgewandelter Dioxystearinsäure erklärt werden, welche, ungeachtet ihrer fast gänzlichen Unlöslichkeit in Äther, bei Anwesenheit des Dibromids in Lösung übergegangen war, oder durch Beimischung von Bromoxystearinsäure, welche das Produkt einer nicht vollständigen Substitution der Hydroxyle der Dioxystearinsäure durch Brom vorstellt. Allem Anschein nach wirken hier beide Ursachen, wie aus der Untersuchung der Produkte nach Abspaltung des Broms hervorgeht. Diese Reaktion wurde ebenso ausgeführt, wie im ersten Versuche; das erhaltene Produkt wurde mit $\mathrm{KOH}$ verseift, die durch HCl ausgeschiedenen Säuren in Äther gelöst, wobei gegen $7 \mathrm{~g}$ in $\ddot{A}$ ther unlöslichen Rückstandes resultierte, welcher, nach seinem Schmelzpunkt zu urteilen, Dioxystearinsäure war.

$\mathrm{Da}$ die nach Verdunsten des Äthers rückständigen Säuren Brom enthielten, so wurde ein Teil des Rückstandes bei $100^{\circ}$ getrocknet und darin Brom bestimmt.

$0,257 \mathrm{~g}$ gaben $0,0235 \mathrm{~g} \mathrm{AgBr}$, daher $\mathrm{Br}=3,89 \%$.

Infolge dieses Umstandes wurde der ganze Rückstand in Alkohol gelöst und von neuem einer Behandlung mit Zinkspänen und Salzsäure unterworfen. Die ausgeschiedenen Säuren wurden nach Verseifen mehrmals aus Petroleumäther, Alkohol und Äther umkristallisiert. Die resultierenden Fraktionen wurden schlieBlich nach ihren Schmelzpunkten in folgende 5 Gruppen eingeteilt.

A) Fraktion vom Schmelzp. $128^{\circ}-130^{\circ}$, ist Dioxystearinsäure, die der Wirkung des Bromwasserstoffs entgangen war.

B) Fraktion vom Schmelzp. 98 -99 $^{\circ}$, Erstarrungspunkt $92^{\circ}-89^{\circ}$, und ihr nahestehend: 1. Schmelzpunkt $94^{\circ}-99^{\circ}$; 2. Schmelzp. $93^{\circ}-97^{\circ}$ und 3. Schmelzp. $93^{\circ}-94^{\circ}$.

Die Schmelzpunkte der verschiedenen Fraktionen dieser Gruppe weisen auf eine Dioxystearinsäure hin, die der Elaidinsäure entspricht. Ihre Entstehung kann leicht erklärt werden durch die Einwirkung von $\mathrm{KOH}$ (bei zweimaligem Verseifen) auf Bromoxystearinsäure.

C) Fraktionen: 1. vom Schmelzpunkt $71^{\circ}-77^{\circ}$; 2. vom Schmelzp. $70^{\circ}-74^{\circ}$, und 3. vom Schmelzp. $69^{\circ}-71^{\circ}$ resultierten in verhältnismäBig nicht großer Quantität.

D) Fraktionen vom Schmelzp. $44^{\circ}-45^{\circ}$, Erstarrungsp. 
294 Albitzky: Kenntnis von Isomerieverhältnisseu etc.

$41^{\circ}-39^{\circ}$ und ihr nahestehend: 1. vom Schmelzp. $41^{\circ}-43^{\circ}$; 2. vom Schmeizp. $40^{\circ}-43^{\circ}$ und 3. vom Schmelzp. $35^{\circ}-41^{\circ}$. Alle diese Fraktionen stellen mehr oder weniger reine Elaidinsäure vor. Daß dieses der Fall ist, läßt sich aus der Untersuchung der Fraktion im ersten Versuche rom ähnlichen Schmelzpunkt ersehen.

E) $13 \mathrm{~g}$ nicht kristallinischer Rückstände, welche unter anderem Ölsäure enthalten. Ihre Anwesenheit lieB sich durch Oxydation der Rückstände mit Kaliumpermanganat in alkalischer Lösung nachweisen. Das Produkt, das nach der Oxydation, aus Alkohol umkristallisiert, gewonnen wurde, schmolz bei $110^{\circ}-114^{\circ}$ und erstarrte bei $104^{\circ}-107^{\circ}$. Aus ihm, nach Umkristallisieren aus $\ddot{A}$ ther, konnte eine Fraktion vom Schmelzpunkt $128^{\circ}-130,5^{\circ}$, Erstarrungsp. $122^{\circ}-116^{\circ}$ ausgeschieden werden. Der letztangeführte Schmelzpunkt deutet auf Dioxystearinsäure hin.

Die zwei oben beschriebenen Versuche zeigen, daß bei der Einwirkung von Bromwasserstoff auf Dioxystearinsäure nicht allein eine Substitution der Hydroxyle durch Brom vor sich geht, sondern dab hierbei ein Teil der anfänglichen Dioxysäure eine Umlagerung in ihre Stereoisomere erfährt, infolge dessen auch zwei Dibromide resultieren, die der Ölund Elaidinsäure entsprechen und aus denen durch Einwirkung von $\mathrm{Zn}$ und $\mathrm{HCl}$ genannte Säuren sich bilden. Die Umlagerung geht während der Bildung des Dibromids vor sich und nicht während der Umwandlung desselben in eine ungesättigte Säure. Aus den Arbeiten von $\mathrm{Burg}^{1}$ ), $\mathrm{Otto}^{2}$ ) und Holt ist bekannt, daB durch Einwirkung von regenerierenden Substanzen auf die Dibromide der Elaidin-, Eruka- und Brassidinsäure wiederum dieselben Säuren resultieren.

Umwandlung der Dioxysäuren in ihre Stereoisomere.

Nachdem die Möglichkeit der Umwandlung der Dioxystearinsäure durch Einwirkung von Bromwasserstoff in die ungesättigte Säure der anderen stereoisomeren Reihe bewiesen

1) Zeitschr. Chem. 1864, S. 718.

2) Ann. Chem. 135, 225.

8) Ber. 24, 4120 (1891). 
worden war, war es von Interesse, eine gegébene Dioxysäure direkt, ohne sie durch die ungesättigte Säure zu führen, in die andere, ihr stereoisomere umzuwandeln. Auf die Möglichkeit einer solchen Umwandlung und den Weg, auf welchem dieses bewerkstelligt werden kann, wies im zweiten beschriebenen Versuche die Bildung einer Fraktion vom Schmelzpunkt $98^{\circ}-99^{\circ}$ hin; zu diesem Zwecke muß nur ein Hydroxyl durch Brom substituiert werden, später aber wird statt dessen wieder ein Hydroxyl eingeführt.

Die Reaktion wurde folgendermafen ausgeführt.

$\mathrm{Zu}$ einer Dioxystearinsäure vom Schmelzp. $133^{\circ}-135^{\circ}$ wurde eine viermal so große Menge Eisessig zugesetzt, und die Mischung bis zum Lösen der Dioxystearinsäure mit Bromwasserstoffgas gesättigt. Am anderen Tage wurde das Reaktionsprodukt durch Wasser zerlegt, das ausgeschiedene Öl durch Filtrieren durch ein nasses Filter von der wäBrigen Flüssigkeit getrennt und mit Wasser ausgewaschen. Das Öl wurde vom Filter durch Äther entfernt. Die Brombestimmung in der öligen Substanz, welche bei langem Stehen im Exsikkator nicht kristallinisch wurde, nachdem sie bei $100^{\circ}$ getrocknet war, ergab folgendes:

1. $0,39 \mathrm{~g}$ gaben $0,1755 \mathrm{~g} \mathrm{AgBr}$.

2. $0,298 \mathrm{~g}$ gaben $0,131 \mathrm{~g} \mathrm{AgBr}$.

Ber. für $\mathrm{C}_{18} \mathrm{H}_{84} \mathrm{Br}\left(\mathrm{C}_{2} \mathrm{H}_{8} \mathrm{O}\right) \mathrm{O}_{2}$ :

Br

18,97

Gefunden:

1. 2 .

$19,1618,71 \%$.

Das Resultat der Analyse zeigt, daß bei der beschriebenen Keaktion eine Substitution eines Hydroxyls durch Brom eingetreten ist, das Wasserstoffatom des anderen aber durch Acetyl ersetzt worden ist; d. h. es hat sich der Acetylester der Bromoxystearinsäure gebildet.

Mit dem erhaltenen Ester wurden folgende Versuche vorgenommen.

$5 \mathrm{~g}$ des Äthers wurden in einer Porzellanschale mit $5 \mathrm{~g}$ starker, wäßriger Lösung von KOH behandelt; dasGemisch wurde unter beständigem Umrühren mit einem Glasstabe auf dem Sandbade solange erwärmt, bis es fast trocken wurde, wonach es in einer Röhre 8 Stunden lang auf $180^{\circ}$ erhitzt wurde. Nach dem Erhitzen wurde der Inhalt der Röhre in eine Schale ge- 
bracht, in Wasser gelöst und durch Salzsäure zerlegt. Die ausgeschiedenen Säuren wurden daraufhin aus Alkohol umkristallisiert. Die erste Fraktion aus Alkohol, umkristallisiert aus Aether, ergab folgende Fraktionen: 1. vom Schmelzpunkt $95^{\circ}-97^{\circ}$, Erstarrungsp. $86^{\circ}-85^{\circ}$ (mehr als ein Gramm); und 2. Schmelzp. $93^{\circ}-96^{\circ}$, Erstarrungsp. $83^{\circ}-81^{\circ}$.

Dieser Versuch wurde unter denselben Bedingungen wiederholt. Die erste Fraktion aus Alkohol, umkristallisiert

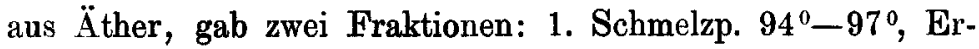
starrungsp. $82^{\circ}-79^{\circ}$ und 2. Schmelzp. $92^{\circ}-93^{\circ}$, Erstarrungspunkt $83^{\circ}-81^{\circ}$.

Die Fraktion vom Schmelzp. $95^{\circ}-97^{\circ}$ ergab bei der Analyse:

$0,1125 \mathrm{~g}$ Substanz gaben $0,283 \mathrm{~g} \mathrm{CO}_{2}$ und $0,117 \mathrm{~g} \mathrm{H}_{2} \mathrm{O}$.

$\begin{array}{ccc} & \text { Berechnet für } \mathrm{C}_{88} \mathrm{H}_{88} \mathrm{O}_{4}: & \text { Gefunden: } \\ \mathrm{C} & 68,27 & 68,61 \% \\ \mathrm{H} & 11,49 & 11,66 \%\end{array}$

Aus den Resultaten der Analyse und den Schmelzpunkten ist zu ersehen, daß die erzielte Substanz Dioxystearinsäure ist; folglich hat sich die anfangs zur Reaktion verwendete Dioxystearinsäure in ihr Stereoisomeres verwandelt. Diese Verwandlung ist während der Bildung des Acetylderivates vor sich gegangen, nicht infolge der Wirkung von $\mathrm{KOH}$ beim Erwärmen; dieses geht aus dem nachfolgenden Versuche der Umwandlung des Monobromoxystearinsäureesters in Dioxystearinsäure hervor; der Versuch wurde unter anderen Bedingungen ausgeführt.

Es ist von mir gezeigt worden ${ }^{1}$ ), daß bei Einwirkung von Ätzbaryt auf Chloroxystearinsäure (das Produkt der Addition von HClO zur Elaidinsäure) eine Glycidsäure erhalten wird, die beim Erwärmen mit Wasser, welches mit Schwefelsäure angesäuert war, Dioxystearinsäure gibt. Eben dieser Weg wurde zur Umwandlung des Essigesters der Monobromoxystearinsäure verwendet.

Für die Reaktion wurden $10 \mathrm{~g}$ Esters im Alkohol gelöst, zur Lösung $15 \mathrm{~g}$ in möglichst geringer Menge Wasser gelösten Ätzbaryts zugesetzt, und das Ganze zum Verseifen 6 Stunden lang auf dem Wasserbade erwärmt. Nach Abdampfen des

1) Dies. Journ. [2] 61, 89 . 
Albitzky: Kenntnis von Isomerieverhältnissen etc. 297

Alkohols wurden die Barytsalze durch Kochen mit HCl zerlegt und die ausgeschiedenen Säuren aus Alkohol umkrystallisiert. Die Krystallisation aus Alkohol ergab $7 \mathbf{g}$; da die erzielte Säure Mineralsalze enthielt, so wurde sie zwecks Reinigung in Eisessig gelöst und aus der Lösung durch Wasser niedergeschlagen. Die abfiltrierte, gewaschene und durch Abpressen zwischen Fließpapier getrocknete Glycidsäure wurde aus $\ddot{A}$ ther umkrystallisiert, wobei folgende Fraktionen resultierten: 1. vom Schmelzp. $54^{\circ}-55^{\circ}$, Erstarrungsp. $40^{\circ}$, ohne Gehalt an Brom und 2. vom Schmelzp. $49^{\circ}-52^{\circ}$, Erstarrungspunkt $29^{\circ}-27^{\circ}$ mit Spuren von Brom.

Die Fraktion vom Schmelzp. 54 $4^{0}-55^{\circ}$ wurde mit Wasser, das durch einige Tropfen Schwefelsäure angesäuert war, in einer zugeschmolzenen Glasröhre 7 Stunden lang auf $160^{\circ}$ erhitzt und dann aus Alkohol umkristallisiert. Die ausgeschiedene Fraktion ergab beim Umkristallisieren aus $\ddot{A}$ ther

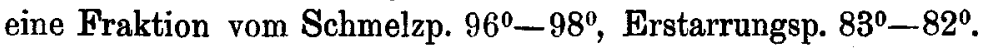
Die alkoholische und ätherische Mutterlauge wurden nach Entfernung der Lösungsmittel mit wäßriger Ätzkalilösung gekocht. Nach der.Zerlegung mittels $\mathrm{HCl}$ und Umkristallisieren aus Alkohol wurde noch wenig Dioxystearinsäure gewonnen, die, nachdem sie auf dem Filter mit Äther ausgewaschen war, bei $95^{\circ}-97^{\circ}$ schmolz und bei $85^{\circ}-83^{\circ}$ erstarrte.

Die Fraktion vom Schmelzp. $49^{\circ}-52^{\circ}$ wurde mit starker wäßriger Kalilauge bearbeitet, das Gemisch getrocknet und in zugeschmolzener Glasröhre 7 Stunden lang auf $160^{\circ}$ erhitzt. Die auf gewöhnlichem Wege ausgeschiedene Säure schmolz, nachdem sie aus Alkohol und Äther umkristallisiert worden war, bei $95^{\circ}-97^{\circ}$ und erstarrte bei $85^{\circ}-83^{\circ}$.

Auf ähnlichem Wege, d. h. dem des Durchganges durch den Essigester der Bromoxybehensäure, wurde Dioxybehensäure vom Schmelzp. $99^{\circ}$ in ihr Stereoisomeres verwandelt.

Zur Bereitung des Esters wurde Dioxybehensäure vom Schmelzp. $96^{\circ}-99^{\circ}$, gewonnen aus Erukasäure durch Oxydation mit dem Ca roschen Reagens, verwendet, die fünffache Menge Eisessig zugesetzt und die Mischung mit Bromwasserstoft gesättigt. $\mathrm{Da}$ ein Löslichwerden der Dioxybehensäure nicht zu bemerken war, so wurde nochmals dieselbe Menge 
Albitzky: Kenntnis von Isomerieverhältnissen etc.

hinzugesetzt, die Mischung von neuem gesättigt und bis zum anderen Tage stehen gelassen. Aber auch am anderen Tage war ein Teil noch ungelöst, daher wurde der Kolben eine Stunde lang auf dem Wasserbade erwärmt, nachher Wasser hinzugesetzt, die ausgeschiedene halbkristallinische Masse abfiltriert, mit Wasser ausgewaschen und schließlich in Äther gelöst. Nach Abdampfen des Äthers verwandelte sich der Ruckstand beim Stehenlassen im Exsikkator in eine kristallinische, mit Ol durchtränkte Masse.

Brombestimmung:

$0,3765 \mathrm{~g}$ Substanz gaben $0,185 \mathrm{~g}$ AgBr.

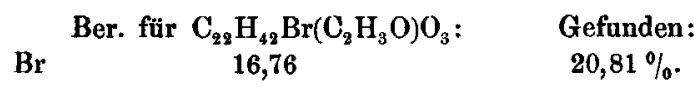

Infolge der unbefriedigenden Resultate wurde die erzielte Substanz einer Reinigung unterworfen. In der Voraussetzung, daß der gewonnene Ester kristallinisch sei, wurde ein Teil desselben auf eine Tonplatte gestrichen, um ihn von den öligen Substanzen zu befreien. lysiert:

Die auf diese Art getrocknete Substanz wurde von neuem ana-

0,274 g Substanz gaben 0,137 g AgBr.

$$
\begin{array}{cc}
\text { Br. für } \mathrm{C}_{22} \mathrm{H}_{42} \mathrm{Br}\left(\mathrm{C}_{2} \mathrm{H}_{3} \mathrm{O}\right) \mathrm{O}_{8}: & \text { Gefunden: } \\
16,76 & 21,28 \% \text {. }
\end{array}
$$

Wie aus obigem zu ersehen ist, waren auch hier die Resultate nicht befriedigend.

Der Mißerfolg bei diesem Versuche konnte dem Umstande zugeschrieben werden, daß hierbei eine Erwärmung der Mischung vorgenommen worden war, bei der der Bromwasserstoff auf den entstandenen Bromoxybehensäureester in der Weise eingewirkt hatte, daB dabei die Acetylgruppe verdrängt wurde. Daher wurde ein anderer Versuch unter veränderten Bedingungen unternommen.

Einer Gewichtsmenge von Dioxybehensäure derselben $\mathbf{A b}$ kunft rom Schmelzp. $96^{\circ}-98^{\circ}$ wurde eine $10 \mathrm{mal}$ so große Gewichtsmenge von Eisessig zugesetzt, die Mischung leicht, bis zum Lösen der Dioxysäure erwärmt und die kaum warme Mischung mit Bromwasserstoff bis zur starken Dampfentwicklung gesättigt. Die bis zum nächsten Tage erkaltete Lösung 
Albitzky: Kenntnis von Isomerieverhältnissen etc. 299 zeigte nar geringen Niederschlag, welcher abfiltriert wurde. Das Filtrat wurde durch Wasser zerlegt, das abgeschiedene Ol abfiltriert, mit Wasser ausgewaschen und durch Äther vom Filter gelöst. Nach Abdampfen des Äthers und Trocknen im Exsikkator wurde ein $\mathrm{Ol}$ gewonnen, das verschwindend wenig kristallinische Substanz enthielt; seine Analyse ergab:

$0,253 \mathrm{~g}$ Substanz gaben $0,09 \mathrm{~g}$ AgBr.

$$
\begin{array}{cc}
\text { Ber. für } \mathrm{C}_{22} \mathrm{H}_{48} \mathrm{Br}\left(\mathrm{C}_{2} \mathrm{H}_{3} \mathrm{O}\right) \mathrm{O}_{3} \text {; } \quad \text { Gefunden: } \\
16,76 & \mathbf{1 5 , 1 2} \% \text {. }
\end{array}
$$

Obgleich die Resultate der Analyse nicht ganz befriedigend waren, so kann doch gesagt werden, daB sich der Essigester gebildet hatte; das Minus an Brom erklärt sich durch die Beimischung jener, in Eisessig nicht löslichen Substanz, die beim Erkalten der Mischung ausgeschieden wurde. Diese Substanz, im Exsikkator getrocknet, wurde auf ibren Gehalt an Brom untersucht.

$0,263 \mathrm{~g}$ Substanz gaben $0,022 \mathrm{~g} \mathrm{AgBr}$.

Daher $\mathrm{Br}=3,56 \%$.

Was diese Substanz war, wurde nicht festgestellt. In keinem Falle kann sie unveränderte Dioxybehensäure sein, da sie leicht in $\ddot{A}$ ther löslich ist (Schmelzp. $73^{\circ}-\mathbf{7 4}^{\circ}$, Erstarrungspunkt $\left.70^{\circ}-69^{\circ}\right)$.

Mit dem gewonnenen Essigester der Bromoxybehensäure wurden folgende Versuche vorgenommen.

$5 \mathrm{~g}$ des Esters wurden mit starker wäßriger Ätzkalilösung behandelt, das Gemisch getrocknet und in zugeschmolzener Glasröhre 6 Stunden lang auf $160^{\circ}$ erhitzt. Die nach dem Erhitzen ausgeschiedenen Säuren gaben nach dem Umkristallisieren aus Alkohol folgende Fraktionen: 1. vom Schmelzp. $122^{\circ}-123^{\circ}$, Erstarrungsp. $113^{\circ}-111^{\circ}$, Gewicht $2 \mathrm{~g}$, und 2. vom Schmelzp. $84^{\circ}-93^{\circ}$, Erstarrungsp. $74^{\circ}-71^{\circ}$. An der ersten Fraktion rom Schmelzp. $122^{\circ}-123^{\circ}$ wurde durch ein zweites Umkristallisieren eine Teilung bewerkstelligt: 1. vom Schmelzpunkt $130^{\circ}-132^{\circ}$, Erstarrungsp. $120^{\circ}$, und 2. Schmelzp. $115^{\circ}$ bis $118^{\circ}$, Erstarrungsp. $106^{\circ}-103^{\circ}$.

Die Analyse der Fraktion vom Schmelzp. $130^{\circ}-132^{\circ}$ ergab folgendes:

$0,1435 \mathrm{~g}$ Substanz gaben $0,374 \mathrm{~g} \mathrm{CO}_{2}$ und $0,1525 \mathrm{~g} \mathrm{H}_{2} \mathrm{O}$. 
300 Albitzky: Kenntnis von Isomerieverhältnissen etc.

$\begin{array}{ccc} & \text { Berechnet für } \mathrm{C}_{22} \mathrm{H}_{44} \mathrm{O}_{4}: & \text { Gefunden: } \\ \mathrm{C} & \mathbf{7 0 , 8 8} & \mathbf{7 1 , 0 7 \%} \\ \mathrm{H} & \mathbf{1 1 , 9 3} & \mathbf{1 1 , 9 1 \%}, .\end{array}$

Die Resultate der Analyse und der Schmelzpunkt beweisen, daB sich hier eine Dioxybehensäure gebildet hat, die der anfangs zur Reaktion verwendeten stereoisomer ist. Eine andere Dioxysäure ist nicht entstanden, wie aus den Schmelztemperaturen der anderen Fraktionen hervorgeht.

Aus dem anderen Teil des Esters wurde Glycidsäure bereitet.

$\mathrm{Zu}$ diesem Zwecke wurden $6 \mathrm{~g}$ des Esters in Alkohol gelöst und unter Erwärmen auf dem Wasserbade 8 Stunden lang mit starker, wäßriger Lösung von $\mathrm{Ba}(\mathrm{OH})_{2}$ verseift. Nach $\mathrm{Ab}$ dampfen des Alkohols wurden die Barytsalze durch Kochen mit Salzsäure zerlegt. Die ausgeschiedenen Säuren wurden dann aus Alkohol umkristallisiert, dem einige Tropfen Eisessig zugesetzt worden waren. Es resultierten $2,5 \mathrm{~g}$ Glycidsäure vom Schmelzp. $68^{\circ}-69^{\circ}$ und Erstarrungsp. $64^{\circ}-63^{\circ}$, sie enthielt Spuren von Brom. Behufs Reinigung wurde die Säure nochmals aus Alkohol umkristallisiert, wonach der Schmelzp. 69" bis $71^{\circ}$ und der Erstarrungsp. $66^{\circ}-65^{\circ}$ war. Brom war nicht mehr vorhanden.

$0,1485 \mathrm{~g}$ Substanz gaben $0,406 \mathrm{~g} \mathrm{CO}_{2}$ und $0,159 \mathrm{~g} \mathrm{H}_{8} \mathrm{O}$.

$\begin{array}{ccc} & \text { Berechnet für } \mathrm{C}_{22} \mathrm{H}_{42} \mathrm{O}_{3} \text { : } & \text { Gefunden: } \\ \mathrm{C} & \mathbf{7 4 , 4 8} & \mathbf{7 4 , 5 6 \%} \\ \mathrm{H} & 11,97 & 12,00 \%\end{array}$

Die analysierte Fraktion vom Schmelzp. $69^{\circ}-71^{\circ}$ wurde in zugeschmolzener Glasröhre mit Wasser, das mit einigen Tropfen schwacher Schwefelsäure angesäuert war, bis $170^{\circ}$ 8 Stunden lang erhitzt. Die nach dem Erhitzen gewonnene Substanz, aus Alkohol umkristallisiert, schied eine Fraktion vom Schmelzp. $126^{\circ}-129^{\circ}$, Erstarrungsp. $119^{\circ}-118^{\circ}$ aus. Die Mutterlauge wurde mit wäBriger Ätzkalilösung verkocht, wonach beim Umkristallisieren der ausgeschiedenen Säure aus Alkohol ein wenig Dioxybehensäure vom Schmelzp. $127^{\circ}-129^{\circ}$, Erstarrungsp. $119^{\circ}-116^{\circ}$, erhalten wurde. 
Albitzky: Kenntnis von Isomerieverhältnissen etc. 301

Aus obigen Versuchen geht hervor, daß der Übergang von einer stereoisomeren Reihe in die andere bei der Substitution der Hydroxyle in den Dioxysäuren durch Brom möglich ist, und $\mathrm{daB}$ diese Umwandlung entweder eine unvollständige - beim Durchgang durch Dibromide, oder eine vollständige, wie zu ersehen war beim Durchgang durch den Essigester der Monobromoxysäuren sein kann. Mit letzterer Folgerung über die vollständige Umwandlung stehen in scheinbarem Widerspruch die Resultate der Untersuchung von Säuren, die bei folgenden Versuchen durch Verseifen vom Diacetylester der Dioxystearinsäure mit Ätzbaryt gewonnen wurden.

Der Diacetylester der Dioxystearinsäure wurde folgendermaßen hergestellt: Ein Essigester der Bromoxystearinsäure, der auf oben beschriebenem Wege zubereitet war, wurde mit essigsaurem Silber gemischt, welches im Überschuß genommen wurde; die Mischung wurde in einer Glasröhre mit einer dem Ester gleichen Menge Eisessig 7 Stunden lang bis $180^{\circ}$ erhitzt.

Nachher wurde der Inhalt der Röhre mit Wasser gekocht, das ausgeschiedene Öl zusammen mit $\mathrm{AgBr}$ abfiltriert und von letzterem durch Lösen in Alkohol getrennt. Ungefähr die Hälfte des Alkohols wurde abdestilliert, zum Rückstande starke wäßrige Lösung von $\mathrm{Ba}(\mathrm{OH})_{2}$ hinzugesetzt und das Ganze auf dem Wasserbade erwärmt. Nach Abdampfen des Alkohols wurden die Barytsalze durch Kochen mit Salzsäure zerlegt und die ausgeschiedenen Säuren zweimal aus Alkohol umkristallisiert. Beim Umkristallisieren aus Äther resultierten Fraktionen: 1. vom Schmelzp. $129^{\circ}-131^{\circ}$, Erstarrungsp. $123^{\circ}-120^{\circ}$; 2. vom Schmelzp. $93^{\circ}-101^{\circ}$, Erstarrungsp. $80,5^{\circ}-78,5^{\circ}$; 3. Schmelzp. $94^{\circ}-96^{\circ}$, Erstarrungsp. $83^{\circ}-79^{\circ}$; 4. Schmelzp. $92^{\circ}-93^{\circ}$, Erstarrungsp. $81^{\circ}-79^{\circ}$, und 5. Schmelzp. $92^{\circ}-95^{\circ}$, Erstarrungsp. $82^{\circ}-79^{\circ}$.

Die erste und zweite Fraktion ergaben bei ihrer Analyse:

1. $0,1289 \mathrm{~g}$ Substanz vom Schmelzp. $129^{\circ}-131^{\circ}$ gaben $0,3226 \mathrm{~g}$ $\mathrm{CO}_{2}$ und $0,1328 \mathrm{~g} \mathrm{H}_{2} \mathrm{O}$.

2. $0,1134 \mathrm{~g}$ Substanz vom Schmelzp. $93^{\circ}-101^{\circ}$ gaben $0,285 \mathrm{~g} \mathrm{CO}_{2}$ und $0,1154 \mathrm{~g} \mathrm{H}_{2} \mathrm{O}$. 
302 Albitzky: Kenntnis von Isomerieverhältnissen etc.

Berechnet für $\mathrm{C}_{19} \mathrm{H}_{88} \mathrm{O}_{4}$ : Gefunden:

$\begin{array}{cccc}\mathrm{C} & 68,27 & 1 . & 2 . \\ \mathrm{H} & 11,49 & 68,25 & 68,54 \% \\ & 11,54 & 11,40 \%\end{array}$

Bei diesem Versuche resultierten zwei Dioxystearinsäuren, wobei die Quantität der höher schmelzenden bedeutend geringer war, als die ihrer Isomeren. Im vorliegenden Falle interessierte die Entstehung der Dioxystearinsäure, die derselben Reihe angehört, wie das Anfangsmaterial zur Bereitung des Essigsäureesters der Bromoxystearinsäure, d. h. der Dioxysäure vom Schmelzp. $129^{\circ}-131^{\circ}$. Ähnliche Fälle der Entstehung normaler Produkte wurden schon früher von mir beim Verseifen des Monoessigesters der Dioxystearinsäuren beobachtet $^{1}$ ), welch letztere durch Einwirkung essigsauren Silbers auf Chloroxystearinsäuren aus Elaidin- und Ölsäure gewonnen worden waren.

Man könnte dieses am wahrscheinlichsten dadurch erklären, daß bei der Bildung des Diacetylesters infolge der Wirkung hoher Temperatur und der Gegenwart von Essigsäure eine umgekehrte Umlagerung erfolgt, d. h. daB die verwendete Dioxystearinsäure von axialsymmetrischer Konfiguration, die bei der Bildung des Essigesters der Bromoxystearinsäure plansymmetrisch geworden war, bei Bildung des Diacetylesters zum Teil wieder in die axialsymmetrische Substanz überging.

Für eine solche Erklärung der Umwandlung durch die gemeinsame Wirkung der Säure und hoher Temperatur spricht auch die Möglichkeit, aus dem Äthylester der $\beta$-Phenylglycerinsäure zwei Dibenzoylester zu erzielen, je nach den Bedingungen der Reaktion. Plöchl und Mayer ${ }^{2}$ ) erhielten bei der Darstellung dieses Esters aus dem Äthylester der $\beta$-Phenylglycerinsäure vom Schmelzp. $141^{\circ}$ nach SchottenBaumann (durch Einwirkung von Benzoylchlorid in alkalischer Lösung bei $0^{\circ}$ ) einen Ester vom Schmelzp. 85 ${ }^{\circ}$, der beim Verseifen wieder dieselbe Säure ergab. Lipp jedoch, der Benzoylchlorid auf den Äthylester derselben Säure bei $150^{\circ}$ ein-

1) Dies. Journ. [2] 61, 86 .

2) Ber. 30, 1606 (1897). 
Albitzky: Kenntnis von Isomerieverhältnissen etc. 303

wirken ließ, erhielt einen Dibenzoylester vom Schmelzp. 109\%, welcher beim Verseifen die andere, $\operatorname{der} \beta$-Phenylglycerinsäure stereoisomere Säure vom Schmelzp. $120^{\circ}-121^{\circ}$ ergab.

Außerdem spricht noch für obige Ansicht der Umstand, $\mathrm{daB}$ wirklich eine nicht vollkommene Umwandlung der Dioxystearinsäuren ineinander beobachtet werden kann, wenn auch nicht beim Erhitzen mit Essigeäure, so doch mit Essigsäureanhydrid, wie aus folgenden Versuchen zu ersehen ist.

Umwandlung der Dioxystearinsäuren in ihre Stereoisomeren durch Einwirkung von Essigsäureanhydrid.

Anfangs wurden Versuche mit Dioxystearinsäure von hohem Schmelzpunkt angestellt; ihr Diacetylester wurde durch Erhitzen im zugeschmolzenen Glasrohr mit Essigsäureanhydrid gewonnen. $\left.{ }^{3}\right)$

$2 \mathrm{~g}$ dieses Esters wurden in Alkohol gelöst und beim Erwärmen auf dem Wasserbade mit starker, wäBriger Ätz. barytlösung verseift. Die mit Hilfe von $\mathrm{HCl}$ nach dem Verseifen und Abdampfen des Alkohols ausgeschiedenen Säuren, aus Alkohol umkrystallisiert, ergaben folgende Fraktionen: 1. vom Schmelzp. $132^{\circ}-135^{\circ}$, Erstarrungsp. $124^{\circ}-120^{\circ}$; 2. Schmelzp. $129^{\circ}-131^{\circ}$, Erstarrungsp. $120^{\circ}-117^{\circ}$, und 3. Schmelzp. $91^{\circ}-102^{\circ}$, Erstarrungsp. $86^{\circ}-78^{\circ}$.

Der Schmelzpunkt der dritten Fraktion deutet auf Bildung von Dioxystearinsäure von niedriger Schmelztemperatur. In verhältnismäBig größerer Quantität wurde sie gewonnen durch Verseifen mit $\mathrm{KOH}$; die Verseifung wurde unter denselben Umständen, die schon öfter beschrieben worden sind, durch Erhitzen im Glasrohr ausgeführt. Beim Umkristallisieren aus Alkohol wurden von den nach der Realtion aus. geschiedenen Säuren zwei Fraktionen erzielt. Die erste wurde mehrmals mit Äther bis zum vollständigen Lösen behandelt.

Die beim Erkalten der ätherischen Filtrate ausgeschiedenen Niederschläge wurden gesammelt, ihr Schmelzpunkt lag bei $128^{\circ}-131^{\circ}$, Erstarrungsp. $122^{\circ}-120^{\circ}$. Beim allmäh-

1) Dies. Journ. [2] 40, 246. 
304 Albitzky: Kenntnis von Isomerieverhältnissen etc.

lichen Abdampfen des Äthers schieden sich folgende Fraktionen aus: 1. vom Schmelzp. $125^{\circ}-130^{\circ} ; 2$. Schmelzp. $106^{\circ}-114^{\circ}$, Erstarrungsp. $106^{\circ}-100^{\circ}$, und 3. Schmelzp. $93^{\circ}-95^{\circ}$, Erstarrungsp. $93^{\circ}-91,5^{\circ}$.

Die zweite Fraktion aus Alkohol, umkristallisiert aus Äther, teilte sich in weitere Fraktionen: 1. Schmelzpunkt $95^{\circ}$ bis $107^{\circ}$, Erstarrungsp. $96^{\circ}-94^{\circ}$; 2. Schmelzp. $94^{\circ}-95^{\circ}$, Erstarrungsp. $93^{\circ}-91^{\circ}$, und 3. Schmelzp. $92^{\circ}-93,5^{\circ}$, Erstarrungspunkt $92^{\circ}-90^{\circ}$.

Noch klarer stellt sich die Entstehung der stereoisomeren Dioxysäure beim Verseifen von Diacetylester, gewonnen aus niedrig schmelzender Dioxystearinsäure (durch Oxydation der Elaidinsäure mit Kaliumpermanganat bereitet). Der Diacetylester, auf gewöhnlichem Wege bereitet, stellt einen dickHüssigen Sirup vor, der sich in seinem Aussehen durch nichts von dem von Spiridon off beschriebenen unterschied.

Seine Verseifung wurde in derselben Weise ausgeführt, wie beim eben beschriebenen Versuche. Durch Umkristallisieren der ausgeschiedenen Säuren aus Alkohol resultierten zwei Fraktionen. Die erste wurde durch Umkristallisieren aus $\ddot{A}$ ther in weitere Fraktionen zerlegt: 1. rom Schmelzp. $128^{\circ}-130^{\circ}$, Erstarrungsp. $120^{\circ}-118^{\circ}$; 2. Schmelzp. $100^{\circ}$ bis $101^{\circ}$, Erstarrungsp. $83^{\circ}-80^{\circ}$, und 3. Schmelzp. $95^{\circ}-97^{\circ}$, Erstarrungsp. $83^{\circ}-78^{\circ}$. Die zweite aus Alkohol, nach Umkrystallisieren aus Äther, zeigte den Schmelzp. $94^{\circ}-98^{\circ}$ und den Erstarrungsp. $80^{\circ}-78^{\circ}$.

Die Fraktion vom Schmelzp. $128^{\circ}-130^{\circ}$ besaß ein Gewicht von $0,5 \mathrm{~g}$, zur Herstellung des Esters waren aber $5 \mathrm{~g}$ $\mathrm{C}_{18} \mathrm{H}_{36} \mathrm{O}_{4}$ rom Schmelzp. $96^{\circ}-98^{\circ}$ verwendet worden.

Ihre Analyse ergab:

0,1575 $\mathrm{g}$ Substanz gaben $0,396 \mathrm{~g} \mathrm{CO}_{2}$ und $0,1675 \mathrm{~g} \mathrm{H}_{2} \mathrm{O}$.

$\begin{array}{ccc} & \text { Berechnet für } \mathrm{C}_{18} \mathrm{H}_{88} \mathrm{O}_{4}: & \text { Gefunden: } \\ \mathrm{C} & 68,27 & 68,57 \% \\ \mathrm{H} & 11,49 & 11,56 \%\end{array}$

Oben beschriebene Versuche der Verseifung waren bei erhöhter Temperatur aus dem Grunde ausgeführt worden, da sie im Anfange vorliegender Untersuchungen vorgenommen wurden, wo noch nicht endgiltig die Ursachen, die eine Um- 
A lbitzky: Kenntnis von Isomerieverhältnissen etc. 305

lagerung veranlassen, erkannt worden waren. Damit weiter kein Zweifel herrsche, daß die Umlagerung während der Bildung des Esters und nicht der Verseifung desselben vor sich geht, wurde folgender Versuch angestellt,

$5 \mathrm{~g}$ einer Dioxystearinsäure vom Schmelzp. $95^{\circ}-97^{\circ}$, gewonnen durch Einwirkung von KOH auf Chloroxystearinsäure aus Ölsäure wurden verwendet. Der aus ihr auf gewöhnlichem Wege bereitete Diacetylester wurde mit alkoholischer Lösung von $\mathrm{KOH}$ unter Erwärmen auf dem Wasserbade verseift. Die ausgeschiedenen Säuren wurden aus Alkohol umkristallisiert. Die erste Fraktion aus Alkohol ergab beim Umkristallisieren aus Äther folgende Fraktionen: 1, vom Schmelzp. $125^{\circ}-127^{\circ}$, Erstarrungsp. $120^{\circ}-118^{\circ}$, Gewicht gegen 0,5 g; 2. Schmelzp. $118^{\circ}-120^{\circ}$; 3. Schmelzp. $111^{\circ}-114^{\circ}$, (2 und 3 sehr wenig), und 4. Schmelzp. $95^{\circ}-97^{\circ}$, Erstarrungsp. $81^{\circ}-80^{\circ}$. Die übrigen Fraktionen wurden nicht untersucht.

Die Fraktion vom Schmelzp. $125^{0}-127^{\circ}$ ergab bei nochmaligem Umkristallisieren aus $\ddot{A}$ ther: 1 , vom Schmelzp. $128^{\circ}$ bis $130^{\circ}$, Erstarrungsp. $125^{\circ}-123^{\circ}$, und 2. Schmelzp. $124^{\circ}$ bis $127^{\circ}$, Erstarrungsp. $122^{\circ}-117^{\circ}$.

Auf diese Weise ist erwiesen, daß infolge der Wirkung des Essigsäureanhydrids unter Erwärmen gleichzeitig mit der Bildung des Diacetylesters der Dioxystearinsäure auch eine teilweise Umlagerung der verwendeten Dioxysäure in ihr Stereoisomers vor sich geht.

\section{Darstellung einer Dioxysäure der anderen stereoisomeren Reibe.}

Die oben beschriebenen Umwandlungen ließen erwarten, daß bei Darstellung von Dioxysäure aus der ungesättigten man diese oder die andere, je nach Wunsch, erhalten kann, wenn man nur die Reaktionsbedingungen ändert. Wie bekannt, resultiert beim Schmelzen mit Silberoxyd, einem alkalischen Reagens, aus dem Dibromid der Ölsäure eine Dioxystearinsäure vom Schmelzp. $136,5^{0}$, aus dem Dibromid der Erukasäure eine Dioxybehensäure vom Schmelzp. $133^{\circ}$; wenn jedoch die Reaktion der Substitution des Broms durch ein Hydroxyl in saurer Lösung geschieht, so können andere ReJournal \&. pralst. Chemie rag $B d$. 62 . 
sultate erwartet werden. Um diese Voraussetzung zu erhärten, wurden Versuche mit Dibromiden der Elaidin-, Eruka- und Brassidinsäure angestellt. Sie wurden in Diacetylester verwandelt und die aus diesen ausgeschiedenen Säuren untersucht.

Zuerst wurden die Versuche mit dem Dibromid der Elaidinsäure ausgeführt. Zur Darstellung des Dibromids wurde zur Lösung der Elaidinsäure vom Erstarrungsp. $45^{\circ}$ in $\mathrm{CCl}_{4}$ tropfenweise eine ebensolche Bromlösung bis zum Entstehen der beständigen Färbung hinzugesetzt, wobei der Kolben, in dem man die Reaktion ausführte, von Zeit zu Zeit durch Wasser abgekühlt wurde. Nach der Verdunstung des größten Teils von $\mathrm{CCl}_{4}$ beim Stehen in einer offenen Schale wurde der Rest durch Erwärmen mit Wasser abgedampft und das Dibromid durch Umkristallisieren aus Äther gereinigt; es zeigte den Schmelzp. $27^{\circ}-29^{\circ}$, und den Erstarrungsp. $15^{\circ}-11^{\circ}$. Das Dibromid der Elaidinsäure schmilzt bei $27^{\circ}$.

Die Brombestimmung ergab:

$0,306 \mathrm{~g}$ Substanz gaben 0,258 g AgBr.

$$
\begin{aligned}
& \text { Berechnet für } \mathrm{C}_{18} \mathrm{H}_{37} \mathrm{Br}_{2} \mathrm{O}_{2} \text { : Gefunden: } \\
& \mathrm{Br} \quad 36,16 \quad 35,88 \% \text {. }
\end{aligned}
$$

Zur Bereitung des Essigesters wurden beim erstenmal $10 \mathrm{~g}$ Dibromid verwendet, die auf dem Wasserbade mit $10 \mathrm{~g}$ (der Theorie gemäB $8 \mathrm{~g}$ ) essigsauren Silbers vermischt, in einer Röhre mit $15 \mathrm{~g}$ Eisessig 7 Stunden lang bis $150^{\circ}$ erbitzt wurden. Nach dem Erhitzen wurde das gewonnene Produkt durch Wasser zerlegt, das ausgeschiedene Öl mit $\mathrm{AgBr}$ abfiltriert, mit Wasser ausgewaschen und von $\mathrm{AgBr}$ durch Auflösen in Äther getrennt. Nach dem Abdampfen des Äthers verblieb als Rückstand ein dickflüssiger Sirup, der eine braune Färbung zeigte. Eine Probe mit Kupferoxyd ergab minimalen Bromgehalt. Ohne vorhergehende Reinigung von unverändertem Dibromid, was, nebenbei gesagt, fast unmöglich ist, wurde der Ester mit starker wäßriger Ätzbarytlösung bei Gegenwart von Alkohol und durch 12 Stunden langes Erwärmen auf dem Wasserbade mit Rückflußkühler verseift. Nach Beendigung der Verseifung und Abdampfen des Alkohols wurden die Barytsalze durch Kochen mit Salzsäure zerlegt und die ausgeschiedenen Säuren aus Alkohol umkristallisiert. 
Albitzky: Kenntnis von Isomerieverhältnissen etc. 307

Die erste Fraktion aus Alkohol, auf dem Filter mit Äther ausgewaschen, wog $4 \mathrm{~g}$ und zeigte' den Schmelzpunkt bei $128^{\circ}-130^{\circ}$, Erstarrungsp. $121^{\circ}-118^{\circ}$. Ein Teil von ihr, für die Analyse aus Äther umkristallisiert, schmolz bei $131^{\circ}$ bis $133^{\circ}$ und erstarrte bei $126^{\circ}-123^{\circ}$. Die zweite Fraktion, die in sehr geringer Menge sich ausschied, schmolz bei $95^{\circ}$ bis $97^{\circ}$ und erstarrte bei $93^{\circ}-91^{\circ}$.

Die Analyse der Fraktion vom Schmelzpunkt $131^{\circ}-133^{\circ}$ ergab folgendes:

$0,132 \mathrm{~g}$ Substanz gaben $0,3295 \mathrm{~g} \mathrm{CO}_{2}$ und $0,1385 \mathrm{~g} \mathrm{H}_{2} \mathrm{O}$.

$\begin{array}{ccc} & \text { Berechnet für } \mathrm{C}_{1 \mathrm{~s}} \mathrm{H}_{36} \mathrm{O}_{4}: & \text { Gefunden: } \\ \mathrm{C} & 68,27 & 68,08 \% \\ \mathrm{H} & 11,49 & 11,76 \%\end{array}$

Ähnliche Resultate, d. h. zwei Dioxystearinsäuren lieferte das Verseifen mit $\mathrm{Ba}(\mathrm{OH})_{2}$ eines Diacetylesters, der aus dem Dibromid durch Erbitzen mit trockenem essigsaurem Natron bei Gegenwart von Essigsäure gewonnen worden war. Dieser Versuch war aus folgenden Gesichtspunkten unternommen worden. Durch Einwirkung von $\mathbf{K O H}$ und $\mathrm{Ba}(\mathrm{OH})_{2}$ auf den Essigsäureester der Bromoxystearinsäure aus Dioxystearinsäure vom Schmelzp. 136,50 erhielt man, wie oben gezeigt wurde, eine Dioxystearinsäure vom Schmelzp. $99^{\circ}$, während, wenn man diesen Ester durch Erbitzen mit essigsaurem Silber in Diacetylester überführt, diesen verseift, beide Stereoisomere resultieren. Es war daher interessant aufzuklären, wovon der Unterschied der beiden Produkte der Reaktion abhängt, ob von einer abermaligen Umlagerung bei der Bildung des Diacetylesters, oder von irgendwelchen specifischen Eigenschaften des Silbers, da nach den Untersuchungen von Walden ein Unterschied in der Wirkung des Silberoxyds oder des Ätzkali und Ätzbaryt zu beobachten ist. ${ }^{\text {I) }}$

Die Bildung des Esters durch Einwirkung von essigsaurem Natron geht bedeutend schlechter vor sich, als mit essigsaurem Silber, wenigstens zeigte die qualitative Probe mit Kupferoxyd bedeutend größeren Gehalt an Brom in dem Ester.

Der Ester wurde mit $\mathrm{Ba}(\mathrm{OH})_{2}$ genau in derselben Weise

1) Ber. 30, 3146 . 
308 Albitzky: Kenntnis von Isomerieverhältnissen etc.

verseift, wie früher. Die aus den Barytsalzen ausgeschiedenen Säuren wurden durch Umkristallisieren aus Alkohol in zwei Fraktionen und Mutterlauge zerlegt.

Die erste Fraktion aus Alkohol nach Umkristallisieren aus Äther ergab: 1. vom Schmelzp. $128^{\circ}-131^{\circ}$, Erstarrungsp. $124^{\circ}-120^{\circ}$, und 2. Schmelzp. $128^{\circ}-131^{\circ}$, Ersarrungsp. $123^{\circ}$ bis $119^{\circ}$.

Die zweite Fraktion aus Alkohol schmolz nach Umkristallisieren aus Äther bei $93^{\circ}-95^{\circ}$ und erstarrte bei $81^{\circ}$ bis $78^{\circ}$. Sie resultierte in bedeutend geringerer Menge.

Also ergiebt sich, daß auch hier dieselben Produlte gewonnen wurden, oder daß die Wirkung des essigsauren Silbers sich qualitativ nicht von der des essigsauren Natrons unterscheidet. Bei der Reaktion des Dibromids mit essigsaurem Nafron bildete sich jedoch, obgleich in nicht großer Menge, Diacetylester, und die ausgeschiedenen Dioxysäuren bildeten sich bei Verseifung auf Kosten des Esters, aber nicht infolge der Einwirkung von $\mathrm{Ba}(\mathrm{OH})_{2}$ auf das Dibromid. In letzterem Falle ging eine Abspaltung des Bromwasserstoffs mit der Bildung von Monobromölsäure vor sich, was durch einen Versuch dargetan wurde.

$5 \mathrm{~g}$ des Dibromids wurden mit $\mathrm{Ba}(\mathrm{OH})_{2}$ erwärmt, wobei alle oben beschriebenen Bedingungen der Reaktion eingehalten wurden. Bei Zerlegung der Barytsalze schied sich ein öl aus, das durch Äther ausgezogen wurde. Nach Abdampfen des Lösungsmittels blieb ein hellgelbes Öl, das trotz halbjährigem Stehen im Exsikkator nicht kristallisierte und stark braun wurde. Die Brombestimmung zeigte, daß die gewonnene Substanz Monobromölsäure war.

0,45 g Substanz gaben 0,227 g AgBr.

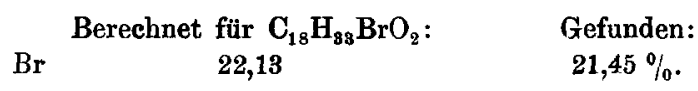

Die Verseifung des aus dem Dtbromid erbaltenen Diacetylesters wurde auch mit Äetzkali unter verschiedenen Bedingungen ausgeführt. Für diese Versuche wurde von neuem aus dem Dibromid der Diacetylester durch Erhitzen mit essigsaurem Silber hergestellt. Da der gewonnene Ester Brom enthielt, so wurde der Gehalt an letzterem bestimmt, um zu 
Albitzky: Kenntnis von Isomerieverhältnissen ete. 309 erkennen, inwieweit die Reaktion des Dibromids mit essigsaurem Silber vollständig verläuft.

$0,33 \mathrm{~g}$ Substanz gaben $0,013 \mathrm{~g} \mathrm{AgBr}$.

$$
\text { Gefunden: } \mathrm{Br}=1,66 \% \text {. }
$$

Bei der neuen Darstellung des Diacetylesters, bei der 8 Stunden lang bis $180^{\circ}$ erhitzt wurde, erzielte man einen Ester, der noch weniger Brom enthielt.

0,289 g Substanz gaben 0,001 g AgBr.

Daher: $\mathrm{Br}=0,15 \%$.

Die Verseifung der gewonnenen Ester wurde zum Teil mit alkoholischer Ätzkalilösung unter Erwärmen auf dem Wasserbade bewirkt. Durch Umkristallisieren der ausgeschiedenen Säuren aus Alkohol resultierten zwei Fraktionen. Die erste, auf dem Filter mit Äther ausgewaschen, schmolz bei $127^{\circ}-129^{\circ}$ und erstarrte bei $120^{\circ}-115^{\circ}$; die zweite schmolz bei $96^{\circ}-103^{\circ}$, beim Umkristallisieren aus Äther gab sie zwei Fraktionen: 1. vom Schmelzpunkt $103^{\circ}-109^{\circ}$ and 2. Schmelzp. $97^{\circ}-99^{\circ}$.

Der andere Teil wurde in einer Schale mit starker wäßriger Ätzkalilösung behandelt, unter beständigem Umrühren die Mischung getrocknet, nachher in Wasser gelöst und durch $\mathrm{HCl}$ zerlegt. Die ausgeschiedenen Säuren ergaben beim Umkristallisieren aus Alkohol zwei Fraktionen. Die erste aus Alkohol, aus Äther umkristallisiert, gab: 1. vom Schmelzp. $129^{\circ}-132^{\circ}$, Erstarrungsp. $123^{\circ}-120^{\circ}$ und 2. Schmelzp. $127^{\circ}$ bis $129^{\circ}$, Erstarrungsp. $126^{\circ}-125^{\circ}$. Die zweite schmolz nach Umkristallisieren aus Äther bei $90^{\circ}-101^{\circ}$ und erstarrte bei $85^{\circ}-83^{\circ}$.

Schließlich wurde im dritten Versuche die Verseifung ebenso vorgenommen wie im zweiten, nur wurde das getrocknete Gemisch in einer zugeschmolzenen Glasröhre sieben Stunden lang bis $160^{\circ}$ erhitzt. Die nach der Reaktion ausgeschiedenen Säuren wurden aus Alkohol umkristallisiert. Das Gemisch derselben wurde nochmals aus Alkohol umkristallisiert, wonach folgende Fraktionen resultierten: 1. Schmelzp. $128^{\circ}-130^{\circ}$, Erstarrungsp. $122^{\circ}-120^{\circ}$, und 2. Schmelzp. $93^{\circ}$ bis $95^{\circ}$, Erstarrungsp. $86^{\circ}-84^{\circ}$. Die erste Mutterlauge ergab 
noch eine Dioxystearinsäure, deren Schmelzpunkt bei $94^{0}-97^{\circ}$, Erstarrungspunkt bei $84^{\circ}-81^{\circ}$ lag.

\section{Erukasäuredibromid.}

Zur Lösung von Erukasäure vom Erstarrungsp. $34^{0}$ in Tetrachlorkohlenstoff wurde allmählich tropfenweise ebensolche Bromlösung zugesetzt. Eine nicht verschwindende Färbung trat auf, bevor noch das berechnete Brom zugegossen worden war. Nachher wurde das Dibromid in eine Schale gegossen, um das $\mathrm{CCl}_{4}$ verdunsten zu lassen, wobei eine nicht beträchtliche Bildung von $\mathrm{HBr}$ erfolgte. Zur Analyse wurde ein Teil des Dibromids bei $100^{\circ}$ getrocknet.

0,3475 g Substanz gaben 0,217 g AgBr.

$$
\begin{array}{ccc} 
& \text { Berechnet für } \mathrm{C}_{22} \mathrm{H}_{42} \mathrm{Br}_{2} \mathrm{O}_{2}: & \text { Gefunden: } \\
\mathrm{Br} & 32,09 & 26,57 \% \text {. }
\end{array}
$$

Aus dem Resultate der Analyse ist zu ersehen, daß das erzielte Dibromid nicht rein war, weshalb es durch Umkristallisieren aus wasserfreiem Äther gereinigt wurde, wobei drei Fraktionen und Mutterlauge resultierten. Mit allen drei Fraktionen wurde eine Brombestimmung vorgenommen.

1. $0,2315 \mathrm{~g}$ der ersten Fraktion gaben $0,0782 \mathrm{~g} \mathrm{AgBr}$.

2. $0,2595 \mathrm{~g}$ der zweiten Fraktion gaben $0,1905 \mathrm{~g} \mathrm{AgBr}$.

3. $0,2624 \mathrm{~g}$ der dritten Fraktion gaben $0,1876 \mathrm{~g} \mathrm{AgBr}$.

Berechnet für $\mathrm{C}_{29} \mathrm{H}_{49} \mathrm{Br}_{2} \mathrm{O}_{2}$ :

Gefunden:

$\mathrm{Br}$

32,09

1. 2.3 . 3. $14,37 \quad 31,24 \quad 30,42 \%$.

Die Analyse zeigt, daß reines Dibromid hauptsächlich in der zweiten Fraktion enthalten ist, welche auch in gröBter Menge resultierte. Ihre Schmelztemperatur war $40^{\circ}-44^{\circ}$, Erstarrungstemperatur $14^{\circ}-13^{\circ}$. Das Erukasäuredibromid. schmilat bei $42^{\circ}-43^{\circ}$. Bei der ersten Fraktion, die bei ungefähr $70^{\circ}$ schmolz, schieden sich gesättigte Säuren aus nach Fileti und Ponzio ${ }^{1}$ ) die Arachinsäure -, die eine beständige Beimischung der Erukasäure sind.

Zur Darstellung des Diacetylesters der Dioxybehensäure wurde nur die zweite Fraktion des Dibromids verwendet. $\mathrm{Zu}$

1) Dies. Journ. [2] 48, 928. 
Albitzky: Kenntnis von Isomerieverhältnissen etc. 311

diesem Zwecke wurde das Dibromid geschmolzen und mit gleicher Menge essigsauren Silbers gemischt, die Mischung in einer Röhre mit Eisessig 8 Stunden lang auf $160^{\circ}$ erhitzt. Nach Beendigung der Reaktion wurde das Produkt durch Wasser zerlegt, das ausgeschiedene Öl zusammen mit $\mathbf{A g B r}$ abfiltriert, mit Wasser ausgewaschen und durch Äther ausgezogen. Der nach dem Abdampfen des Äthers zurückgebliebene Diacetylester war ein dickflüssiges braungefärbtes Öl. Die Probe mit Kupferoxyd wies einen geringen Gehalt an Brom nach, welcher bestimmt wurde.

$0,25 \mathrm{~g}$ Substanz gaben 0,003 g AgBr.

Gefunden: $\quad B r=0,51 \%$.

Mit dem gewonnenen Ester wurden folgende Versuche angestellt: Die Verseifung mit Ätzbaryt wurde in derselben Weise ausgeführt, wie schon öfter beschrieben (12 stündiges Erhitzen). Die ausgeschiedenen Säuren schmolzen nach zweimaligem Umkristallisieren aus geringer Menge Alkohol und Auswaschen auf dem Filter mit Äther bei $107^{\circ}-112^{\circ}$ und erstarrten bei $99^{\circ}-91^{\circ}$. Die Schmelztemperatur zeigt, daB man es hier mit einem Gemisch von zwei Dioxybehensäuren zu tun hatte.

Zum Zweck ihrer Trennung wurde das Gemisch aus großer Menge von Alkohol umkristallisiert, wobei zwei Fraktionen resultierten: 1 . Schmelzp. $110^{\circ}-116^{\circ}$, Erstarrungsp. $104^{\circ}-101^{\circ}$, und 2. Schmelzp. $98^{\circ}-102^{\circ}$, Erstarrungsp. $89^{\circ}$ bis $87^{\circ}$. Die erste Fraktion ergab nach nochmaligem Umkristallisieren aus viel Alkohol ihrerseits zwei Fraktionen: 1. Schmelzp. $122^{\circ}-125^{\circ}$, Erstarrungsp. $114^{\circ}-112^{\circ}$, und 2. Schmelzp. $96^{\circ}-100^{\circ}$, Erstarrungsp. $90^{\circ}-88^{\circ}$. A ußerdem wurde aus dem Gemisch der zwei letzten Mutterlaugen eine Fraktion vom Schmelzp. $96^{\circ}-98^{\circ}$, Erstarrungsp. $88^{\circ}-87^{\circ}$ abgeschieden. Aus den Schmelztemperaturen der ausgeschiedenen Fraktionen ist zu ersehen, daB beide Dioxybehensäuren erhalten worden waren.

1. $0,1186 \mathrm{~g}$ Substanz vom Schmelzp. $98^{\circ}-102^{\circ}$ gaben $0,3066 \mathrm{~g}$ $\mathrm{CO}_{2}$ und $0,1265 \mathrm{~g} \mathrm{H}_{2} \mathrm{O}$.

2. $0,1649 \mathrm{~g}$ Substanz vom Schmelzp. $122^{\circ}-125^{\circ}$ gaben $0,4276 \mathrm{~g}$ $\mathrm{CO}_{2}$ und $0,1836 \mathrm{~g} \mathrm{H}_{2} \mathrm{O}$. 
312 Albitzky: Kenntnis von Isomerieverhältnissen etc.

\begin{tabular}{cccc} 
& Berechnet für $\mathrm{C}_{22} \mathrm{H}_{44} \mathrm{O}_{4}:$ & \multicolumn{2}{c}{ Gefunden: } \\
& & 1. & 2. \\
$\mathrm{C}$ & 70,88 & 70,50 & $70,71 \%$ \\
$\mathrm{H}$ & 11,93 & 11,95 & $12,48 \%$.
\end{tabular}

Nachher wurde noch eine Verseifung mit alkoholischer Lösung von KOH unter Erwärmen auf dem Wasserbade ausgeführt. Die auf gewöhnlichem Wege ausgeschiedenen Säuren, aus wenig Alkohol umkristallisiert, zeigten den Schmelzpunkt $98^{\circ}-105^{\circ}$. Nach Umkristallisieren aus viel Alkohol resultierten zwei Fraktionen: 1. vom Schmelzp. $119^{\circ}-121^{\circ}$, Erstarrungsp. $109^{\circ}-106^{\circ}$, und 2. Schmelzp. $96^{\circ}-98^{\circ}$, Erstarrungspunkt $86^{\circ}-85^{\circ}$.

Die Analyse der Fraktion vom Schmelzp. $95^{\circ}-98^{\circ}$ ergab:

$0,131 \mathrm{~g}$ Substanz gaben 0,341 g $\mathrm{CO}_{2}$ und $0,142 \mathrm{~g} \mathrm{H}_{2} \mathrm{O}$.

$\begin{array}{ccc} & \text { Berechnet für } \mathrm{C}_{28} \mathrm{H}_{44} \mathrm{O}_{4}: & \text { Gefunden: } \\ \mathrm{C} & 70,88 & \mathbf{7 0 , 9 9 \%} \% \\ \mathrm{H} & 11,93 & 12,14 \% .\end{array}$

Schließlich wurde noch der Ester mit starker wäßriger KOH-Lösung behandelt und die Mischung in einer Röhre 7 Stunden lang bis $160^{\circ}$ erhitzt. Die auf gewöhnlichem Wege ausgeschiedenen Säuren schmolzen nach zweimaligem Umkristallisieren aus wenig Alkohol bei $107^{\circ}-110^{\circ}$ und erstarrten bei $102^{\circ}-96^{\circ}$. Umkristallisieren aus viel Alkohol ergab zwei Fraktionen: 1. Schmelzp. $112^{0}-115^{0}$, Erstarrungsp. $106^{\circ}-102^{\circ}$, und 2. Schmelzp. $97^{\circ}-99^{\circ}$, Erstarrungsp. $90^{\circ}-89^{\circ}$. Die erste von diesen zuletzt erhaltenen Fraktionen wurde ihrerseits geteilt in: 1. Schmelzp. $124^{\circ}-126^{\circ}$, Erstarrungsp. $117^{\circ}-114^{\circ}$, und 2. Schmelzp. $100^{\circ}-103^{\circ}$, Erstarrungsp. $89^{\circ}-87,5^{\circ}$. AuBerdem konnte aus der Mischung der zwei letzten Mutterlaugen noch eine Fraktion vom Schmelzp. 97 $-98^{\circ}$, Erstarrungsp. $90^{\circ}-89^{\circ}$ abgeschieden werden.

Hieraus ist ersichtlich, daB aus Diacetylester der Dioxybehensäure, gewonnen aus dem Dibromid der Erukasäure, beim Verseifen immer zwei Dioxybehensäuren resultieren.

Das Brassidinsäuredibromid wurde auf dieselbe Weise, wie die vorhergehenden Dibromide dargestellt. Zur Lösung der Brassidinsäure vom Erstarrungsp. $60^{\circ}$ in Tetrachlorkohlenstoff wurde tropfenweise ebensolche Lösung von Brom bis zum Erscheinen der nicht verschwindenden Färbung zugesetzt. 
Albitzky: Kenntnis von Isomerieverhältnissen etc. 313

Nach Verdunsten des größten Teils des Lösungsmittels wurde der Rest durch Erwärmen auf dem Wasserbade entfernt. Das gewonnene Dibromid, das einer Reinigung durch Umkristallisieren nicht unterworfen worden war, schmolz bei $49^{\circ}$ bis $52^{\circ}$. Das Brassidinsäuredibromid schmilat bei $54^{\circ}$.

Eine Brombestimmung ergab:

0,274 g Substanz gaben $0,197 \mathrm{~g} \mathrm{AgBr}$.

$$
\begin{aligned}
& \text { Berechnet für } \mathrm{C}_{22} \mathrm{H}_{42} \mathrm{Br}_{2} \mathrm{O}_{2} \text { : Gefunden: } \\
& \mathrm{Br} \quad 32,09 \quad 30,59 \% \text {. }
\end{aligned}
$$

Da hiernach das Dibromid sich als ziemlich rein erwies, so wurde es direkt, ohne vorhergehendes Umkristallisieren, in den Diacetylester der Dioxybehensäure verwandelt, zu welchem $Z_{\text {wecke es }}$ geschmolzen und mit einer gleichen Gewichtsmenge essigsauren Silbers gemischt wurde. Die Mischung wurde in einer Röhre mit Eisessig 8 Stunden lang bis $160^{\circ}$ erhitzt. Das Produkt der Reaktion wurde durch Wasser zerlegt, der in Form von Öl ausgeschiedene Ester zusammen mit $\mathbf{A g B r}$ abfiltriert und von letzterem durch Lösen in Äther getrennt. Eine Probe mit Kupferoxyd zeigte die Anwesenheit ron Brom, dessen Menge daraufhin bestimmt wurde.

0,286 g Substanz gaben 0,011 g AgBr.

$$
\text { Gefunden: } \mathrm{Br}=1,65 \% \text {. }
$$

Mit diesem Ester wurden die nachfolgenden Versuche der Verseifung ausgeführt: Zuerst wurde er mit Ätzbaryt in der schon bekannten Weise verseift. Der Schmelzpunkt der so gewonnenen Säuren, nachdem sie aus wenig Alkohol umkristallisiert waren, lag bei $107^{\circ}-115^{\circ}$, der Erstarrungspunkt bei $100^{\circ}-95^{\circ}$. Dann ergab ein Umkristallisieren aus viel Alkohol Fraktionen: 1. vom Schmelzp. $120^{\circ}-122^{\circ}$, Erstarrungspunkt $113^{\circ}-108^{\circ} ; 2$. Schmelzp. $97^{\circ}-100^{\circ}$, Erstarrungsp. $86^{\circ}$ bis $84^{\circ}$; 3. Schmelzp. $97^{\circ}-100^{\circ}$, Erstarrungsp. $85^{\circ}-84^{\circ}$, und 4. Schmelzp. $97^{\circ}-100^{\circ}$, Erstarrungsp. $94^{\circ}-82^{\circ}$. Aus der ersten Fraktion wurde zum Zweck der Analyse eine Fraktion vom Schmelzp. $128^{\circ}-130^{\circ}$, Erstarrungsp. $119^{\circ}-117^{\circ}$ ausgeschieden; aus der 2., 3. und 4. Fraktion aber, die vereinigt wurden, zu demselben Zwecke eine Fraktion vom Schmelzp. 97,5 $5^{\circ}-99,5^{\circ}$, Erstarrungsp. $88^{\circ}-86^{\circ}$ gewonnen.

1. $0,1385 \mathrm{~g}$ Substanz vom Schmelzp. $97,5^{\circ}-99,5^{\circ}$ gaben $0,361 \mathrm{~g}$ $\mathrm{CO}_{2}$ und $0,1505 \mathrm{~g} \mathrm{H}_{2} \mathrm{O}$. 
314 Albitzky: Kenntnis von Isomerieverhältnissen etc.

2. $0,1385 \mathrm{~g}$ Substanz vom Schmelzp. $128^{\circ}-130^{\circ}$ gaben $0,36 \mathrm{~g} \mathrm{CO}_{2}$ und $0,15 \mathrm{~g} \mathrm{H}_{2} \mathrm{O}$.

\begin{tabular}{cccc} 
& Berechnet für $\mathrm{C}_{22} \mathrm{H}_{44} \mathrm{O}_{4}:$ & \multicolumn{2}{c}{ Gefunden: } \\
$\mathrm{C}$ & & 1. & 2. \\
$\mathrm{H}$ & 70,88 & 71,08 & $70,88 \%$ \\
& 11,93 & 12,18 & $12,14 \%$.
\end{tabular}

Ein zweiter Versuch der Verseifung wurde mit alkoholischer Lösung von Ätzkali unter Erwärmen auf dem Wasserbade ausgeführt. Beim Umkristallisieren der auf gewöhnlichem Wege erhaltenen Säuren aus Alkohol resultierten zwei Fraktionen: 1. vom Schmelzp. $116^{\circ}-120^{\circ}$, Erstarrungsp. $99^{\circ}-94^{\circ}$, und 2. Schmelzp. $98^{\circ}-100^{\circ}$. Bei nochmals vorgenommenem Umkristallisieren beider Fraktionen stellte sich der Schmelzpunkt der ersten höher, bei $124^{\circ}-126^{\circ}$ ebenso der Erstarrungsp. $113^{0}-108^{\circ}$; bei der zweiten jedoch zeigten beide Punkte keine Änderung, da sie $98^{\circ}-100^{\circ}$ und $86^{\circ}-84^{\circ}$ verblieben.

Schließlich wurde ein dritter Versuch folgendermaßen ausgeführt. Der Ester wurde mit starker wäBriger $\mathrm{KOH}$ Lösung behandelt, die Mischung getrocknet und 7 Stunden lang in zugeschmolzenem Rohr bis auf $160^{\circ}$ erhitzt. Die Säuren schmolzen nach dem Umkristallisieren aus wenig Alkohol bei $108^{\circ}-116^{\circ}$ und erstarrten bei $94^{\circ}-86^{\circ}$, aus viel Alkohol gaben sie folgende Fraktionen: 1. vom Schmelzpunkt $112^{\circ}-116^{\circ}$, Erstarrungsp. $96^{\circ}-90^{\circ} ; 2$. Schmelzp. $102^{\circ}-106^{\circ}$, Erstarrungsp. $87^{\circ}-85^{\circ} ; 3$. Schmelzp. $94^{\circ}-96^{\circ}$, Erstarrungsp. $86^{\circ}-84^{\circ}$.

Die Fraktion vom Schmelzp. $112^{\circ}-116^{\circ}$ zerfiel ihrerseits beim Umkristallisieren in zwei: 1 . Schmelzp. $111^{\circ}-117^{\circ}$, Erstarrungsp. $102^{\circ}-98^{\circ}$, und 2. Schmelzp. $94^{\circ}-97^{\circ}$, Erstarrungspunkt $85^{\circ}-82^{\circ}$. SchlieBlich resultierten aus der Fraktion vom Schmelzp. $111^{\circ}-117^{\circ}$ auch zwei: 1. vom Schmelzpunkt $127^{\circ}-129^{\circ}$, Erstarrungsp. $116^{\circ}$, und 2. Schmelzp. $103^{\circ}-107^{\circ}$, Erstarrungsp. $91^{\circ}-87^{\circ}$.

Aus diesen Versuchen kann gefolgert werden, daß auch beim Verseifen des Diacetylesters der Dioxybehensäure, gewonnen aus Brassidinsäuredibromid, immer beide stereoisomer Dioxybehensäuren resultieren.

Die Entstehung anormaler Produkte bei beschricbener Reaktion wird durch eine Umlagerung bedingt, die während 
Walbaum u. Hüthig: Kenntnis des Neroliöles etc. $\mathbf{3 1 5}$

der Wechselwirkung des Dibromids und essigsauren Salzes vor sich geht. Die Reaktion der Addition von Brom zu den höheren ungesättigten Säuren erfolgt anders, als bei den Krotonsäuren, der Tiglin- und Angelikasäure, wo gewöhnlich zwei Dibromide gebildet werden. Aus den Arbeiten von Holt ist bekannt, daB bei der Addition von Chlor oder Brom eine Umlagerung nicht erfolgt, da beim Abspalten des Haloids dieselben Säuren resultieren, die anfangs zur Reaktion verwendet worden sind.

\section{Beiträge znr Kenntnis des Neroliöles und Petit- grainöles; \\ von}

H. Walbaum und $\mathrm{O}$. Hüthig.

(Mitteilung aus dem Laboratorium von Sehimmel und Co. in Miltitz bei Leipzig.)

\section{I. Über Neroliöl.}

In einer im Dezember vorigen Jahres erschienenen Abhandlung über das Orangenblütenöl ${ }^{1}$ ) bemerken A. Hesse und $O$. Zeitschel, indem sie auf eine von uns 'herrührende Arbeit Bezug nehmen, folgendes: „Im Begriff,' die Resultate unserer Untersuchung der Öffentlichkeit zu übergeben, erfahren wir aus dem letzten Geschäftsbericht (Oktober 1902) der Firma Schimmel u. Co., daB eine Publikation über die in dem Laboratorium dieser Firma gemachten Untersuchungen des Neroliöls zu erwarten ist. In ihrer vorläufigen Mitteilung sagt genannte Firma, daß der $\mathrm{Nach}$ eis von Pinen, Kamphen, Dipenten, 1-Linalool und d-Terpineol gelungen ist, und $\mathrm{da} B$ die Gegenwart eines Aldehyds, wahrscheinlich Decylaldehyds,

1) Dies. Journ. [2] 66, 481 (1902). 